\title{
Helium and deuterium abundances as a test for the time variation of the fine structure constant and the Higgs vacuum expectation value
}

\author{
N Chamoun ${ }^{1,2}$, S J Landau ${ }^{3,4,5}$, M E Mosquera ${ }^{3,6}$ and Hector Vucetich ${ }^{1,3}$ \\ ${ }^{1}$ Departamento de Física, Universidad Nacional de La Plata, cc67, 1900 La Plata, Argentina \\ 2 Physics Department, HIAST, PO Box 31983, Damascus, Syria \\ ${ }^{3}$ Facultad de Ciencias Astronómicas y Geofísicas, Universidad Nacional de La Plata, \\ Paseo del Bosque, cp 1900 La Plata, Argentina \\ ${ }^{4}$ Departamento de Física, FCEyN, Universidad de Buenos Aires, Ciudad Universitaria-Pab. 1, \\ 1428 Buenos Aires, Argentina \\ E-mail: nidal@venus.fisica.unlp.edu.ar, slandau@df.uba.ar and mmosquera@fcaglp.unlp.edu.ar
}

Received 7 July 2006

Published 4 December 2006

Online at stacks.iop.org/JPhysG/34/163

\begin{abstract}
We use the semi-analytic method of Esmailzadeh et al (1991 Astrophys. J. 378 504-18) to calculate the abundances of helium and deuterium produced during Big Bang nucleosynthesis assuming the fine structure constant and the Higgs vacuum expectation value may vary in time. We analyse the dependence on the fundamental constants of the nucleon mass, nuclear binding energies and cross sections involved in the calculation of the abundances. Unlike previous works, we do not assume the chiral limit of QCD. Rather, we take into account the quark masses and consider the one-pion exchange potential, within perturbation theory, for the proton-neutron scattering. However, we do not consider the time variation of the strong interactions scale but attribute the changes in the quark masses to the temporal variation of the Higgs vacuum expectation value. Using the observational data of the helium and deuterium, we put constraints on the variation of the fundamental constants between the time of nucleosynthesis and the present time.
\end{abstract}

\section{Introduction}

Big Bang nucleosynthesis (BBN) offers the deepest reliable probe of the early universe. Predictions of the abundances of the light elements $\mathrm{D},{ }^{3} \mathrm{He},{ }^{4} \mathrm{He}$ and ${ }^{7} \mathrm{Li}$ synthesized at the end of the 'first three minutes' are in good overall agreement with the primordial abundances

5 Member of the Carrera del Investigador Científico y Tecnológico, CONICET.

${ }^{6}$ Fellow of CONICET. 
inferred from observational data, which validates the standard Big Bang nucleosynthesis (SBBN). BBN also provides powerful constraints on possible deviations from the standard cosmology and on new theories on physics beyond the standard model (SM) (Sarkar 1996). Among these theories, there are those in which some of the dimensionless ratios of fundamental constants do vary in time such as string-derived field theories (Wu and Wang 1986, Maeda 1988, Barr and Mohapatra 1988, Damour and Polyakov 1994, Damour et al 2002a, 2002b), related brane-world theories (Youm 2001a, 2001b, Palma et al 2003, Brax et al 2003) and (related or not) Kaluza-Klein theories (Kaluza 1921, Klein 1926, Weinberg 1983, Gleiser and Taylor 1985, Overduin and Wesson 1997). On the other hand, recent astronomical data (Webb et al 1999, 2001, Murphy et al 2001a, 2001b, 2003) suggest a possible variation of the fine structure constant $\alpha=e^{2} / \hbar c$ at the $10^{-5}$ level over a time-scale of 10 billion years. However, other recent independent analysis of similar data (Martínez Fiorenzano et al 2003, Quast et al 2004, Bahcall et al 2004, Srianand et al 2004, Grupe et al 2005, Chand et al 2006) found no variation. On the other hand, measurements of molecular hydrogen (Ivanchik et al $2002,2003,2005)$ reported a variation of the proton to electron mass $\mu=\frac{m_{p}}{m_{e}}$. This fact motivated more general discussions of possible variations of other constants. Langacker $e t$ al (2002) have studied the implication of gauge unification for the time variation of $\alpha$ while Olive et al (2002) explored a super-symmetric version of the dynamical Bekenstein model (Bekenstein 1982) in order to produce a large change in $\alpha$ in the redshift range $z=0.5-3.5$ and still be consistent with the constraints on $\Delta \alpha / \alpha$ from the results of high precision limits on the violation of equivalence principle by a fifth force.

On the other hand, there are many non-SBBN models which introduce new free parameters in addition to the baryon density parameter, or equivalently the baryon asymmetry $\eta_{B} \equiv \frac{n_{B}-n_{\bar{B}}}{n_{\gamma}}=2.74 \times 10^{-8} \Omega_{B} h^{2}$. Most known of these models are those which assume either a non-standard contribution to the total density, or a lepton asymmetry. The first possibility affects the expansion rate of the universe $S \equiv \frac{H^{\prime}}{H}=\sqrt{\frac{\rho^{\prime}}{\rho}}$ and can be restated in terms of 'equivalent' number of extra neutrinos $\Delta N_{v}=N_{v}-3$. Simple analytic fits to BBN and the cosmic microwave background radiation (CMBR) data provide the following bound: $0.85<S<1.15$ (Barger et al 2003a, 2003b, Steigman 2005, 2006). As regards the lepton asymmetry, observational data do not imply that should be connected to the 'tiny' baryon asymmetry $\eta_{B}$, and it could be large enough to perturbe SBBN predictions . Moreover, a small asymmetry between electron type neutrinos and antineutrinos can have a significant impact on BBN since the $v_{e}$ affect the interconversion of neutrons to protons changing the equilibrium neutron-to-proton ratio from $(n / p)_{e q}^{0}=e^{-\frac{\Delta m}{T}}$ to $(n / p)_{e q}=(n / p)_{e q}^{0} e^{-\xi_{e}}$. In consequence, the ${ }^{4} \mathrm{He}$ abundance changes. In contrast the $\mathrm{D}$ abundance is insensitive to $\xi_{e} \neq 0$. Consistent with the BBN and CMBR data, values of $\xi_{e}$ in the range $-0.1<\xi_{e}<0.3$ are permitted (Barger et al 2003a, Steigman 2005, 2006). In our analysis, however, we shall not consider these non-SBBN scenarios, but attribute any non-SBBN issue to time variation of fundamental constants.

The density of baryonic matter $\Omega_{B} h^{2}$ can be estimated using the WMAP data from the CMBR (Spergel et al 2003, 2006). From the observed WMAP baryon density, the predicted abundances are highly consistent with the observed D but not with ${ }^{4} \mathrm{He}$ and ${ }^{7} \mathrm{Li}$. However, any change in the value of the fundamental constants would work its way into the value of the abundances of the various light elements and the question we address is whether or not existing observations of the primordial abundances suggest any change in the values of the fundamental constants at the time of BBN.

$\mathrm{BBN}$ is sensitive to a number of fundamental dimensionless parameters including the fine structure constant $\alpha, \Lambda_{\mathrm{QCD}} / M_{\text {Plank }}$ and $m^{q} / \Lambda_{\mathrm{QCD}}$ where $m^{q}$ is the quark mass and $\Lambda_{\mathrm{QCD}}$ 
is the strong scale determined by the position of the pole in the perturbative QCD running coupling constant. Several authors have studied the dependences of the BBN parameters on the fundamental constants. The dependence of the primordial abundances on the fine structure constant has been evaluated by Bergström et al (1999) and improved by Nollett and Lopez (2002). Yoo and Scherrer (2003) analyse the effects of the time variation of the Higgs vacuum expectation value $\langle v\rangle$ on $\mathrm{BBN}$ and the cosmic microwave background radiation (CMBR). On the other hand, Müller et al (2004) calculate the abundances as a function of the Planck mass $M_{P}, \alpha,\langle v\rangle$, electron mass $m_{e}$, nucleon decay time, deuterium binding energy $\left(\epsilon_{D}\right)$ and neutron-proton mass difference $\left(\Delta m=m_{n}-m_{p}\right)$. Moreover, they study the dependence of the last three quantities as functions of the fundamental couplings and masses. Kneller and McLaughlin (2003) study the dependence of the primordial abundances on $\epsilon_{D}$ and $\Delta m$. These papers (Yoo and Scherrer 2003, Müller et al 2004, Kneller and McLaughlin 2003) use the results of chiral perturbation theory (Beane and Savage 2003) to address the dependence of the deuterium binding energy with the Higgs vacuum expectations value and $\Lambda_{\mathrm{QCD}}$. However, the dependence of the deuterium binding energy $\epsilon_{D}$ on $\langle v\rangle$ was estimated from an approximated linear dependence of $\epsilon_{D}$ on the pion mass $m_{\pi}$, while the exact limits on the relative change of $\langle v\rangle$ would depend on the details of such dependence. On the other hand, Campbell and Olive (1995) and Ichikawa and Kawasaki (2002) study the effects of variation of fundamental constants on BBN in the context of a dilaton superstring model. Finally, limits on cosmological variations of $\alpha, \Lambda_{\mathrm{QCD}}$ and $m^{q}$ from optical quasar absorption spectra, laboratory atomic clocks and from BBN have been established by Flambaum and Shuryak (2002) and Flambaum et al (2004). For computing the deuterium binding energy $\left(\epsilon_{D} \approx 2.225 \mathrm{MeV}\right)$ they apply quantum mechanics perturbation theory. This factor is very significant in influencing the reaction rate of $p+n \rightarrow d+\gamma$ which is the first and most crucial step in BBN.

The BBN abundances can be computed using numerical (Wagoner 1973, Kawano 1992) and analytical (Esmailzadeh et al 1991, Mukhanov 2003) methods. In a previous work (Landau et al 2006), we used the semi-analytic method of Esmailzadeh et al (1991) to calculate the abundances of the light elements produced during BBN assuming that the gauge coupling constants may vary in time. We considered the chiral limit of QCD when analysing the nucleon masses, binding energies and the cross sections. Deviations between standard cosmology calculations and observational data could be interpreted as resulting from variations in $G_{F}$ the Fermi constant, $\alpha$ the fine structure constant and $\Lambda_{\mathrm{QCD}}$ the strong interactions scale. The semi-analytical method allows us to obtain semi-analytic dependences of the primordial abundances on the fundamental constants, which otherwise must be computed using numerical codes.

On the other hand, in the standard model, a variation of the Fermi constant implies a variation of the vacuum expectation value (vev) of the Higgs field (Dixit and Sher 1988):

$$
G_{F}=\frac{\alpha_{\text {weak }}\left(M_{W}\right)}{\sqrt{2} M_{W}^{2}}=\frac{1}{\sqrt{2}\langle v\rangle^{2}} .
$$

Here $M_{W}$ is the mass of the $W$-boson, and $\langle v\rangle \approx 250 \mathrm{GeV}$ is the vev of the Higgs field. Within the QCD chiral limit, the quark masses, which are also proportional to the Higgs vev through the relation $m^{q}=Y_{\text {Yukawa }}\langle v\rangle$, are neglected. Therefore, the logical and consistent step to follow is to go beyond the chiral limit and take the variation of $\langle v\rangle$ as affecting the Fermi constant as well as the quark masses. We analyse the nucleon masses, the nuclear binding energies and the cross sections dependence within quantum mechanics perturbation theory. The objective of this paper is to study such variations as model independent as possible. Therefore, we consider the one-pion exchange potential as the perturbation on the 
p-n scattering responsible for the formation of the deuterium. This perturbation potential varies in time if the pion mass changes in time which leads to a time variation of the deuterium binding energy. The pion mass also depends on the Higgs vev through the Gell-Mann-OakesRenner relation: $m_{\pi}^{2}=m^{q} \frac{|\langle\bar{q} q\rangle|}{f_{\pi}^{2}} \simeq m^{q} \Lambda_{\mathrm{QCD}}$, where $\langle\bar{q} q\rangle$ is the quark condensate and $f_{\pi}$ is the pion decay constant. In order to determine the dependence of the deuterium binding energy on the fundamental constants, we use the square well model to approximate the attractive strong interaction potential of the deuterium and fit current scattering data to get estimates for the depth and width of the well. On the other hand, we will not discuss the effect of $\Lambda_{\mathrm{QCD}}$ variation on the QCD-determined quantities such as the quark condensate or the width and depth of the square well. The reason for this is that we lack a complete theory for these quantities, and, especially, because of the absence of p-n scattering data in the far past. For the same reason, we will not consider changes in the Yukawa couplings either. On the other hand, the effect of $\Lambda_{\mathrm{QCD}}$ variation on the abundances of the light elements was analysed in a previous work (Landau et al 2006). Thus, we will limit ourselves in this paper to studying the dependence on $\alpha$ and $\langle v\rangle$ for the physical quantities, such as binding energies, nucleon masses and cross sections involved in the BBN calculations. Our treatment of the deuterium binding energy is similar to the one performed by of Flambaum and Shuryak (2002). However, there are some technical differences in the wavefunction normalization which we describe in section 4. Furthermore, we go one step further in calculating the effects of such variations in the fundamental constants on the primordial abundances of D and ${ }^{4} \mathrm{He}$. This is one of the advantages of using the semi-analytical method and it also allows us to compare with observational data in order to put bounds on the variation of $\alpha$ and $\langle v\rangle$.

On the other hand, the concordance between the WMAP estimates and SBBN has been investigated by many authors (Cyburt et al 2003, Romano et al 2003, Cuoco et al 2004, Cyburt 2004, Coc et al 2004a, 2004b). From the WMAP baryon density, the predicted abundances are highly consistent with the observed D but not with ${ }^{4} \mathrm{He}$ and ${ }^{7} \mathrm{Li}$. They are produced more than observed. Such discrepancy is usually ascribed to non-reported systematic errors in the observations of ${ }^{4} \mathrm{He}$ and ${ }^{7} \mathrm{Li}$. Indeed, more realistic determinations of the ${ }^{4} \mathrm{He}$ uncertainty implies a baryon density in line with the WMAP estimate (Cyburt 2004, Olive and Skillman 2004). On the other hand, Richard et al (2005) have pointed out that a better understanding of turbulent transport in the radiative zones of the stars is needed for a better determination of the ${ }^{7} \mathrm{Li}$ abundance. In our previous work, we obtained results consistent with variation of fundamental constants when considering all data. However, discarding the ${ }^{7} \mathrm{Li}$ data we obtained no variation. Therefore, we suspect that the possible non-reported systematic uncertainties are 'hidden' within a setup involving variation of the fundamental constants. Thus, until better estimations of the systematic errors of ${ }^{7} \mathrm{Li}$ are reported, we will only consider the $\mathrm{D}$ and ${ }^{4} \mathrm{He}$ data.

Even though the WMAP estimate of the baryon density is the most accurate one, it is still affected by degeneracies with other cosmological parameters (Spergel et al 2003, 2006). On the other hand, this quantity can be also determined combining data from galaxy surveys (SDSS, 2dF) and X-ray satellites (Chandra, XMM-Newton, ROSAT, ASCA) (Landau et al 2006). In this work, we consider a weighted mean between the WMAP estimate and Landau et al (2006) estimate for $\Omega_{b} h^{2}$, and, furthermore, we shall compute the dependence of binding energies, cross sections and abundances on this parameter. Finally, we shall use observational data from $\mathrm{D}$ and ${ }^{4} \mathrm{He}$ to estimate the variations in time of $\alpha$ and $\langle v\rangle$ and a possible deviation of $\Omega_{b} h^{2}$ from its considered value. We also compare our results with other non-SBBN models, where a non-standard expansion rate and an electron-neutrino asymmetry were considered. Finally, we would like to emphasize that the approach in this work is phenomenological and the results we get are model independent. 
Table 1. Stages and equations. $n$ refers to neutron, $p$ to proton, $d$ to deuterium, $T$ to tritium, 3 to ${ }^{3} \mathrm{He}$ and $\alpha$ to ${ }^{4} \mathrm{He}$.

\begin{tabular}{lll}
\hline Stage & Equations & Final temperature \\
\hline Until the weak interaction freeze-out & & \\
Until the production of ${ }^{4}$ He becomes efficient & $\dot{Y}_{n}=-2 \dot{Y}_{\alpha}-Y_{n}[n]$ & $2 \dot{Y}_{\alpha} \sim Y_{n}[n]$ \\
& $\begin{array}{l}\dot{Y}_{d}=\dot{Y}_{3}=\dot{Y}_{T}=0 \\
\dot{Y}_{n}=-2 \dot{Y}_{\alpha}\end{array}$ & $Y_{n}=Y_{d}$ \\
Until the production of deuterium dominates & $\begin{array}{l}\dot{Y}_{d}=\dot{Y}_{3}=\dot{Y}_{T}=0 \\
\text { rate of change of neutrons }\end{array}$ & $\begin{array}{l}\dot{Y}_{d}=-2 \dot{Y}_{\alpha} \\
\text { Deuterium final abundance } \\
\dot{Y}_{n}=\dot{Y}_{3}=\dot{Y}_{T}=0\end{array}$ \\
\end{tabular}

The paper is organized as follows. In section 2 we present the notations used and summarize the steps which one follows in the semi-analytic approach to calculate the abundances. In section 3 we calculate the dependence of the abundances on $\alpha,\langle v\rangle, \Omega_{b} h^{2}$ and the deuterium binding energy $\epsilon_{D}$. In section 4 , we express the dependence of the deuterium binding energy on the Higgs vev within the square well model. Results of comparing theoretical prediction with observational values are presented in section 5, where we also compare with other non-standard BBN models results. Conclusions are presented in section 6 .

\section{Preliminaries}

The method of Esmailzadeh et al (1991) consists of calculating the different abundances between fixed points or stages. One solves the equations for the light elements only for one element in each stage. For the other elements (say, the $i$ th), it is necessary to solve the quasistatic equilibrium (QSE) equation $\left(\dot{Y}_{i} \approx 0\right)$, where $Y_{i}$ is the abundance of the $i$ th element relatively to baryons, considering only the most important rates of production and destruction. On the other hand, we should also calculate the final temperature of each stage. We show in table 1 the different stages and their corresponding equations, to which is added also the conservation of the neutron number (further details are given in Esmailzadeh et al (1991) and Landau et al (2006)).

Since we are considering changes in the Higgs vev $(\langle v\rangle)$ and the fine structure constant $(\alpha)$, we need to find expressions for the nucleon masses and binding energies in terms of these quantities. For the P-N mass splitting we have

$$
Q=\Delta m=m_{n}-m_{p}=\Delta^{\alpha} m+\Delta^{\rho-w} m,
$$

where $\Delta^{\alpha} m$ is the contribution of the electromagnetic energy, and thus $\frac{\delta \Delta^{\alpha} m}{\Delta^{\alpha} m}=\frac{\delta \alpha}{\alpha}$. On the other hand, $\Delta^{\rho-w} m$ is due to $\rho-w$ mesons mixing and known to be proportional to $\frac{m_{s}^{2}}{m_{u}+m_{d}}$ (Christiansen et al 1991). Therefore, $\frac{\delta \Delta^{\rho-w} m}{\Delta^{\rho-w} m}=\frac{\delta m^{q}}{m^{q}}=\frac{\delta\langle v\rangle}{\langle v\rangle}$. Thus, we get

$$
\frac{\delta Q}{Q}=-0.587 \frac{\delta \alpha}{\alpha}+1.587 \frac{\delta\langle v\rangle}{\langle v\rangle} .
$$

We need also to know the dependence of the variation of the nuclear mass of an element ${ }_{Z}^{A} X$ in terms of the changes in $\langle v\rangle$ and $\alpha$. This can be estimated using $M(X)=Z m_{p}+N m_{n}-\epsilon_{X}$ where $\epsilon_{X}$ is the binding energy for the element $X$, and we have

$$
\frac{\delta \epsilon_{x}}{\epsilon_{x}}=\frac{\epsilon_{C}}{\epsilon_{x}} \frac{\delta \alpha}{\alpha},
$$


where $\epsilon_{C}=\frac{Z}{4 \pi \epsilon_{0}} \frac{e^{2}}{R}$ is the electromagnetic contribution. The radius of the nucleus $(R \sim$ $1.2 A^{\frac{1}{3}} \mathrm{fm}$ ) is considered as a strong interaction effect and, thus, taken to be constant in our analysis. The change in the neutron decay rate in terms of the changes in $\alpha$ and $\langle v\rangle$ can be expressed as follows (Ichikawa and Kawasaki 2002, Landau et al 2006):

$$
\frac{\delta \tau}{\tau}=-3.838 \frac{\delta \alpha}{\alpha}-4.793 \frac{\delta\langle v\rangle}{\langle v\rangle},
$$

where we have used $\frac{\delta G_{F}}{G_{F}}=-2 \frac{\delta\langle v\rangle}{\langle v\rangle}$. For the thermonuclear reaction rates dependence on $\alpha$, we take the phenomenological expressions of tables 4 and 5 in Landau et al (2006).

Since BBN is very sensitive to $\epsilon_{D}$, we should go further from equation (4) to evaluate the changes in $\epsilon_{D}$ in terms of $\delta\langle v\rangle$. We will give our expressions for the different stages in terms of $\frac{\delta\langle v\rangle}{\langle v\rangle}, \frac{\delta \epsilon_{D}}{\epsilon_{D}}, \frac{\delta \alpha}{\alpha}$ and $\frac{\delta \Omega_{B} h^{2}}{\Omega_{B} h^{2}}$. In section 4 we find an estimate for $\frac{\delta \epsilon_{D}}{\epsilon_{D}}$ in terms of $\frac{\delta\langle v\rangle}{\langle v\rangle}$, and thus we can give then the final expressions in terms of $\frac{\delta\langle v\rangle}{\langle v\rangle}, \frac{\delta \alpha}{\alpha}$ and $\frac{\delta \Omega_{B} h^{2}}{\Omega_{B} h^{2}}$.

\section{Abundances and their dependence on $\alpha$ and $\langle v\rangle$ in the different stages}

The ratio $X$ of the number of neutrons to the total number of baryons in the first stage until the freeze-out of weak interactions $\left(T>9.1 \times 10^{9} \mathrm{~K}\right.$ ) can be expressed as follows (Bernstein et al 1989):

$$
X(y=\infty)=\int_{0}^{\infty} \mathrm{d} y^{\prime} e^{y^{\prime}} \frac{1}{1+e^{y}}\left(y^{\prime}\right)^{2} e^{-K\left(y^{\prime}\right)}=0.151,
$$

where $K(y)=b\left[\frac{4}{y^{3}}+\frac{3}{y^{2}}+\frac{1}{y}+\left(\frac{4}{y^{3}}+\frac{1}{y^{2}}\right) e^{-y}\right]$. Only $b=255 \frac{M_{p l}}{\Delta m^{2} \tau} \sqrt{\frac{45}{43 \pi^{3}}}$ depends on the fundamental constants through $\tau$ and $\Delta m$, so we get

$$
\frac{\delta X(y=\infty)}{X(y=\infty)}=-0.52 \frac{\delta b}{b} \text {. }
$$

Using equations (3) and (5) we obtain

$$
\frac{\delta X(y=\infty)}{X(y=\infty)}=1.385 \frac{\delta \alpha}{\alpha}-0.842 \frac{\delta\langle v\rangle}{\langle v\rangle} .
$$

In the second stage, after weak interactions freeze out, neutrons decay freely until the rate of production of ${ }^{4} \mathrm{He}$ becomes efficient $\left(9.1 \times 10^{9} \mathrm{~K}>T>0.93 \times 10^{9} \mathrm{~K}\right)$. Thus we have

$$
Y_{n}=X(y=\infty) e^{-t / \tau}=0.151 e^{-0.2 / T_{9}^{2}},
$$

where $T_{9}$ is the temperature evaluated in units of $10^{9} \mathrm{~K}$.

The abundance of deuterium follows its equilibrium value and we assume the reactions $[n p \mathrm{~d} \gamma]$ and $[\mathrm{d} \gamma n p]$ dominate for its production and destruction. Taking $\Omega_{B} h^{2}=0.0223$ we can calculate the final temperature of this stage by setting $\dot{Y}_{n}=0$ and thus $2 \dot{Y}_{n}=-Y_{n}[n]$. We find $T_{9}^{f}=0.93$ and get the abundances $Y_{p}=0.76$ and $Y_{n}=0.12$. In order to calculate the dependence of the final temperature on the fundamental constants, we derive the equation $2 \dot{Y}_{n}=-Y_{n}[n]$ with respect to the fundamental constants to get

$$
\frac{\delta T_{9}}{T_{9}}=0.065 \frac{\delta \Omega_{B} h^{2}}{\Omega_{B} h^{2}}+0.055 \frac{\delta \alpha}{\alpha}-0.119 \frac{\delta\langle v\rangle}{\langle v\rangle}+1.195 \frac{\delta \epsilon_{D}}{\epsilon_{D}},
$$

and thus we get the relative variations of the nucleons abundances for this stage as follows:

$$
\begin{aligned}
\frac{\delta Y_{n}}{Y_{n}} & =0.030 \frac{\delta \Omega_{B} h^{2}}{\Omega_{B} h^{2}}+2.159 \frac{\delta \alpha}{\alpha}-2.005 \frac{\delta\langle v\rangle}{\langle v\rangle}+0.553 \frac{\delta \epsilon_{D}}{\epsilon_{D}} \\
\frac{\delta Y_{p}}{Y_{p}} & =-0.009 \frac{\delta \Omega_{B} h^{2}}{\Omega_{B} h^{2}}-0.682 \frac{\delta \alpha}{\alpha}+0.634 \frac{\delta\langle v\rangle}{\langle v\rangle}-0.174 \frac{\delta \epsilon_{D}}{\epsilon_{D}} .
\end{aligned}
$$


In order to compute the final abundance of helium, we notice that once ${ }^{4} \mathrm{He}$ production becomes efficient (i.e., $2 \dot{Y}_{\alpha}=Y_{n}[n]$ ), neutrons combine to form $\alpha$-particles, and the production of the latter is dominated by $[d T n \alpha]$ and $[p T \gamma \alpha]$. One gets for the temperature of the ${ }^{4} \mathrm{He}$ freeze-out the following equation:

$$
2 Y_{n}\left(Y_{p} \frac{[n p \mathrm{~d} \gamma]}{Y_{\gamma}[\mathrm{d} \gamma n p]}\right)^{2}[d d p T]=\frac{1}{\tau},
$$

where $Y_{p}=0.76, Y_{n}=0.151 e^{-0.2 / T_{9}^{2}}$ and $\tau$ is the neutron decay constant. Numerically we find $T_{9}^{\alpha}=0.915$ which is lower than the final temperature of the previous stage and larger than the final temperature of the next one. For the final helium abundance we find $Y_{\alpha}^{f}=2 Y_{n}=0.238$. As before, deriving equation (10) with respect to $\epsilon_{D},\langle v\rangle$ and $\alpha$ we find

$$
\frac{\delta T_{9}^{\alpha}}{T_{9}^{\alpha}}=0.061 \frac{\delta \Omega_{B} h^{2}}{\Omega_{B} h^{2}}+0.049 \frac{\delta \alpha}{\alpha}-0.113 \frac{\delta\langle v\rangle}{\langle v\rangle}+1.149 \frac{\delta \epsilon_{D}}{\epsilon_{D}} .
$$

Since $Y_{\alpha}^{f}=2 Y_{n}$ we get the relative variation of the helium abundance as

$$
\frac{\delta Y_{\alpha}^{f}}{Y_{\alpha}^{f}}=0.029 \frac{\delta \Omega_{B} h^{2}}{\Omega_{B} h^{2}}+2.182 \frac{\delta \alpha}{\alpha}-2.042 \frac{\delta\langle v\rangle}{\langle v\rangle}+0.549 \frac{\delta \epsilon_{D}}{\epsilon_{D}} .
$$

In the following 'neutron cooking' stage, corresponding to $0.93 \times 10^{9} \mathrm{~K}>T>$ $0.766 \times 10^{9} \mathrm{~K}$, the neutron abundance can be expressed as follows:

$$
Y_{n}=\left(\frac{1}{Y_{n}^{0}}+2 \int_{t_{\text {initial }}}^{t}\left(Y_{p} \frac{[n p \mathrm{~d} \gamma]}{Y_{\gamma}[\mathrm{d} \gamma n p]}\right)^{2}[d d p T] \mathrm{d} t\right)^{-1},
$$

where the initial condition is given by the final values of the previous stage: $Y_{n}^{0}=0.12$ at $T_{9}^{0}=0.93$.

Putting $Y_{n}=Y_{d}$ as the condition which determines the final temperature of this stage, we find $\frac{[n p \mathrm{~d} \gamma]}{Y_{\gamma}[\mathrm{d} \gamma n p]}=1$. With $Y_{p}$ freezed at 0.76 , we get numerically

$$
\begin{aligned}
& T_{9}^{f}=0.766 \\
& Y_{n}=6.4 \times 10^{-4}=Y_{d} .
\end{aligned}
$$

Again, the condition $\left(Y_{n}=Y_{d}\right)$ allows the calculation of the relative change of the final temperature:

$$
\frac{\delta T_{9}^{f}}{T_{9}^{f}}=0.031 \frac{\delta \Omega_{B} h^{2}}{\Omega_{B} h^{2}}-0.021 \frac{\delta \alpha}{\alpha}+0.020 \frac{\delta\langle v\rangle}{\langle v\rangle}+1.041 \frac{\delta \epsilon_{D}}{\epsilon_{D}}
$$

and we get numerically

$$
\frac{\delta Y_{d}}{Y_{d}}=\frac{\delta Y_{n}}{Y_{n}}=-1.095 \frac{\delta \Omega_{B} h^{2}}{\Omega_{B} h^{2}}+1.865 \frac{\delta \alpha}{\alpha}-0.075 \frac{\delta\langle v\rangle}{\langle v\rangle}-2.275 \frac{\delta \epsilon_{D}}{\epsilon_{D}} .
$$

In the last stage $\left(T<0.766 \times 10^{9} \mathrm{~K}\right)$, we note that the dominant term in the time derivative of $Y_{d}$ is the production of tritium, i.e. $Y_{d} Y_{d}[d d p T]$. We find

$$
Y_{d}=\left(\frac{1}{Y_{d}^{0}}+2 \int_{t_{\text {initial }}}^{t}[d d p T] \mathrm{d} t\right)^{-1}
$$

with the initial value $Y_{d}^{0}=6.4 \times 10^{-4}$ at $T_{9}^{0}=0.766$. 
Table 2. Final abundances and their relative variations in terms of the relative variations of the fundamental constants, $\frac{\delta Y_{i}^{f}}{Y_{i}^{f}}=A \frac{\Omega_{B} h^{2}}{\Omega_{B} h^{2}}+B \frac{\delta \alpha}{\alpha}+C \frac{\delta\langle v\rangle}{\langle v\rangle}+D \frac{\delta \epsilon_{D}}{\epsilon_{D}}$.

\begin{tabular}{lrllrl}
\hline$Y_{i}^{f}$ & \multicolumn{1}{c}{$A$} & $B$ & $C$ & \multicolumn{1}{c}{$D$} & Abundance \\
\hline$D$ & -1.072 & 2.318 & -0.049 & -2.469 & $2.41 \times 10^{-5}$ \\
${ }^{4} \mathrm{He}$ & 0.029 & 2.182 & -2.042 & 0.549 & 0.238 \\
\hline
\end{tabular}

We can obtain the final abundance of deuterium by setting the temperature equal to zero and we find the abundance numerically equal to $Y_{d}^{f}=2.41 \times 10^{-5}$. Again, we can numerically evaluate the relative change of $Y_{d}^{f}$ :

$$
\frac{\delta Y_{d}^{f}}{Y_{d}^{f}}=-1.072 \frac{\delta \Omega_{B} h^{2}}{\Omega_{B} h^{2}}+2.318 \frac{\delta \alpha}{\alpha}-0.049 \frac{\delta\langle v\rangle}{\langle v\rangle}-2.469 \frac{\delta \epsilon_{D}}{\epsilon_{D}} .
$$

We summarize the results that we obtained in table 2 .

\section{The dependence of deuterium binding energy $\epsilon_{D}$ on the Higgs vev $\langle v\rangle$}

As we said before, the deuterium binding energy $\epsilon_{D}$ is the most significant factor that can influence the BBN reactions rates, and its variation was discussed in Flambaum and Shuryak (2002, 2003), Dmitriev and Flambaum (2003) and Dmitriev et al (2004). Indeed, the equilibrium concentration of deuterons and the inverse reaction rate depend exponentially on it. Moreover, the deuterium is a shallow bound level $\left(\epsilon_{D} \approx 2.225 \mathrm{MeV}\right)$. Therefore the relative variation of the deuterium binding energy $\epsilon_{D}$ is much larger than the relative variation of the strong interaction potential which we neglect in our work. In order to give an estimate for the relative variation of $\epsilon_{D}$, we should compute, within perturbation theory, the correction to $\epsilon_{D}$ due to the perturbation which might change in time. Thus we write $\epsilon_{D}=\epsilon_{D}^{0}+\Delta E$, where $\epsilon_{D}^{0}$ is the unperturbed binding energy and we consider it a QCD-determined quantity which does not change in time. As to $\Delta E$, we know (Weinberg 1990, 1991) that the onepion exchange potential represents the first approximation to the perturbation on the strong interaction potential, and it has the form:

$$
V^{Y}=\frac{f^{2}}{4 \pi} \frac{e^{-m_{\pi} r}}{r}
$$

where $\frac{f^{2}}{4 \pi} \sim 0.08, m_{\pi} \sim 140 \mathrm{MeV}$ is the pion mass. We simplify the strong interaction potential by a square well model with width $a$ and depth $V_{0}$. These two parameters can be determined by fitting the square well 'theoretical' expressions involving these two parameters to the p-n scattering data. According to the shape-independent effective range theory (Bethe 1949, Bethe and Longmire 1950) all the binding and low energy scattering properties of the potential are determined by just two parameters which can be determined experimentally: the scattering length $a_{t}=5.50 \times 10^{-13} \mathrm{~cm}$ and the effective range $r_{t}=1.72 \times 10^{-13} \mathrm{~cm}$ (Schiff 1968). The corresponding values for the square well are the depth $V_{0}=35.5 \mathrm{MeV}$ and the width $a=2.03 \times 10^{-13} \mathrm{~cm}=0.0103 \mathrm{MeV}^{-1}$. We consider the width and the depth as QCD-determined parameters and assume they do not change in time.

Now, we have in the square well model

$$
\Delta E=\frac{f^{2} A^{2}}{4 \pi \xi^{2}} \int_{0}^{\xi a} \frac{\sin ^{2} x}{x} \mathrm{e}^{-\frac{m_{\pi}}{\xi} x} \mathrm{~d} x+\frac{f^{2} B^{2}}{4 \pi \beta^{2}} \int_{\xi a}^{\infty} \frac{\mathrm{e}^{-\left(\frac{m_{\pi}}{\xi}+\frac{2 \beta}{\xi}\right) x}}{x} \mathrm{~d} x,
$$


where

$$
\begin{aligned}
& \xi=\sqrt{m_{N}\left(V_{0}-\epsilon_{D}\right)}=176.76 \mathrm{MeV} \\
& \beta=\sqrt{m_{N} \epsilon_{D}}=45.71 \mathrm{MeV} \\
& A=\sqrt{\frac{\xi^{2}}{\frac{a}{2}-\frac{\sin (2 \xi a)}{4 \xi}+\frac{\sin ^{2}(\xi a)}{2 \beta}}}=1393 \mathrm{MeV}^{3 / 2} \\
& B=-\frac{\beta}{\xi} \sin (\xi a) e^{\beta a} A=-559 \mathrm{MeV}^{3 / 2}
\end{aligned}
$$

and $m_{N}$ is the reduced mass of the two-nucleon system.

Whence,

$$
\Delta E=0.203 \mathrm{MeV} \text {. }
$$

On the other hand, the change due to the variation of the nucleon mass in the Yukawa potential $V^{Y}$ is negligible compared with the change due to the variation of $m_{\pi}$. Since $\frac{\delta m_{\pi}}{m_{\pi}}=\frac{1}{2} \frac{\delta\langle v\rangle}{\langle v\rangle}$ as mentioned before, we get, evaluating numerically the integrals, the following:

$$
\frac{\delta \Delta E}{\Delta E}=-0.896 \frac{\delta m_{\pi}}{m_{\pi}}=-0.448 \frac{\delta\langle v\rangle}{\langle v\rangle},
$$

and so

$$
\frac{\delta \epsilon_{D}}{\epsilon_{D}}=-0.041 \frac{\delta\langle v\rangle}{\langle v\rangle} .
$$

These values are one order of magnitude lower than those obtained by Flambaum and Shuryak (2002). The difference arises from the fact that these authors did not consider the continuity of the wavefunction and its derivative on the boundary of the square well. This results in differences between the normalization factor of the wavefunctions which propagate into the binding energy first order perturbation. Moreover, the values of $a$ and $V_{0}$ they consider are different from this work.

Hence, the final expressions for the relative variations of the helium and deuterium abundances are

$$
\begin{aligned}
\frac{\delta Y_{d}^{f}}{Y_{d}^{f}} & =-1.072 \frac{\delta \Omega_{B} h^{2}}{\Omega_{B} h^{2}}+2.318 \frac{\delta \alpha}{\alpha}+0.052 \frac{\delta\langle v\rangle}{\langle v\rangle} \\
\frac{\delta Y_{\alpha}^{f}}{Y_{\alpha}^{f}} & =0.029 \frac{\delta \Omega_{B} h^{2}}{\Omega_{B} h^{2}}+2.182 \frac{\delta \alpha}{\alpha}-2.044 \frac{\delta\langle v\rangle}{\langle v\rangle} .
\end{aligned}
$$

These results are summarized in table 3

\section{Results}

We can now compare the theoretical predictions of the abundances of ${ }^{4} \mathrm{He}$ and $\mathrm{D}$ obtained in the last section with the observational data. The equations (24), (25) are of the form $\left(i=1(\mathrm{D}), 2\left({ }^{4} \mathrm{He}\right)\right)$ :

$$
\frac{\delta Y_{i}^{f}}{Y_{i}^{f}}=A_{i} \frac{\delta \Omega_{B} h^{2}}{\Omega_{B} h^{2}}+B_{i} \frac{\delta \alpha}{\alpha}+C_{i} \frac{\delta\langle v\rangle}{\langle v\rangle}
$$


Table 3. Abundances and their dependence on fundamental constants, $\frac{\delta Y_{i}^{f}}{Y_{i}^{f}}=A \frac{\delta \alpha}{\alpha}+B \frac{\delta\langle v\rangle}{\langle v\rangle}+$ $C \frac{\delta \Omega_{B} h^{2}}{\Omega_{B} h^{2}}$.

\begin{tabular}{lllrr}
\hline$Y_{i}^{f}$ & Abundance & $A$ & \multicolumn{1}{c}{$B$} & \multicolumn{1}{c}{$C$} \\
\hline${ }^{2} \mathrm{H}$ & $2.41 \times 10^{-5}$ & 2.318 & 0.052 & -1.072 \\
${ }^{4} \mathrm{He}$ & 0.238 & 2.182 & -2.044 & 0.029 \\
\hline
\end{tabular}

Table 4. Theoretical abundances in the standard model with the WMAP estimate $\Omega_{B} h^{2}=0.0223$.

\begin{tabular}{ll}
\hline Nucleus & $Y_{i}^{\text {SBBN }} \pm \delta Y_{i}^{\text {SBBN }}$ \\
\hline${ }^{2} \mathrm{H}$ & $(2.51 \pm 0.37) \times 10^{-5}$ \\
${ }^{4} \mathrm{He}$ & $0.2483 \pm 0.0021$ \\
\hline
\end{tabular}

Table 5. Observational abundances.

\begin{tabular}{lll}
\hline Nucleus & $Y_{i}^{\text {obs }} \pm \delta Y_{i}^{\text {obs }}$ & Reference \\
\hline $\mathrm{D}$ & $(1.65 \pm 0.35) \times 10^{-5}$ & (Pettini and Bowen 2001) \\
$\mathrm{D}$ & $(2.54 \pm 0.23) \times 10^{-5}$ & (O'Meara et al 2001) \\
$\mathrm{D}$ & $\left(2.42_{-0.25}^{+0.35}\right) \times 10^{-5}$ & (Kirkman et al 2003) \\
$\mathrm{D}$ & $(3.25 \pm 0.3) \times 10^{-5}$ & (Burles and Tytler 1998a) \\
$\mathrm{D}$ & $\left(3.98_{-0.67}^{+0.59}\right) \times 10^{-5}$ & (Burles and Tytler 1998b) \\
$\mathrm{D}$ & $\left(1.6_{-0.30}^{+0.25}\right) \times 10^{-5}$ & (Crighton et al 2004) \\
${ }^{4} \mathrm{He}$ & $0.244 \pm 0.002$ & (Izotov and Thuan 1998) \\
${ }^{4} \mathrm{He}$ & $0.243 \pm 0.003$ & (Izotov et al 1997) \\
${ }^{4} \mathrm{He}$ & $0.2345 \pm 0.0026$ & (Peimbert et al 2000) \\
${ }^{4} \mathrm{He}$ & $0.232 \pm 0.003$ & (Olive and Steigman 1995) \\
\end{tabular}

and we take the assumption that the difference $\frac{\delta Y_{i}^{f}}{Y_{i}^{f}}$ is due to a change in the considered fundamental constants: $\frac{\delta Y_{i}}{Y_{i}}=\frac{Y_{i}^{\mathrm{obs}}-Y_{i}^{\mathrm{SBBN}}}{Y_{i}^{\mathrm{SBBN}}}$, where $Y_{i}^{\mathrm{SBBN}}$ and $Y_{i}^{\mathrm{obs}}$ are the theoretical and observed abundances, respectively. In table 4, the theoretical abundances $Y_{i}^{\mathrm{SBBN}}$ are given for $\Omega_{B} h^{2}=0.0223$ with their errors resulting from the uncertainty in the values of the parameters involved. In table 5, the observational data of helium and deuterium are stated with their measured errors.

As regards the consistency of the $\mathrm{D}$ and ${ }^{4} \mathrm{He}$ data, we follow the treatment of (Landau et al 2006) and increase the observational error by a factor $\Theta$. The values of $\Theta$ are 2.4 for $\mathrm{D}$ and 2.33 for ${ }^{4} \mathrm{He}$.

The results of solving the system of equations (26) with the given data are shown in table 6 . These results are consistent within $1-\sigma$ with no variation of the fundamental constants. On the other hand, the results considering variation of one fundamental constant only are shown in table 7. These results are consistent within $3-\sigma$ with no variation of the fundamental constants.

In order to check the goodness of our fit, we performed a Kolmogorov-Smirnov (KS) test (see figure 1). When considering variation in $\Omega_{B} h^{2}, \alpha$ and $\langle v\rangle$ altogether, we have a 


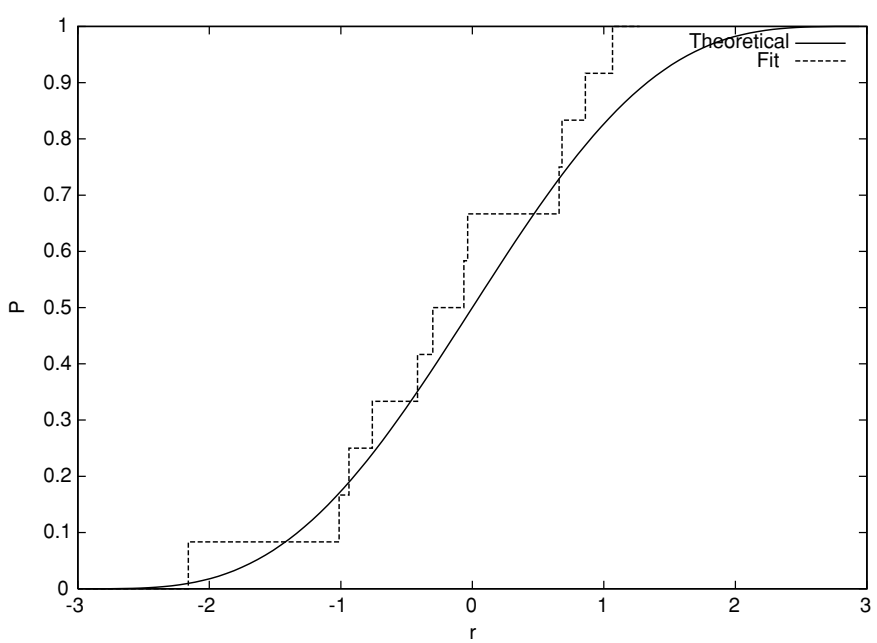

Figure 1. The full line shows the theoretical probability of the residuals, while the dotted line shows the empirical probability. Deviations of $\Omega_{B} h^{2}, \alpha$ and $\langle v\rangle$ with respect to their mean values are considered.

Table 6. Results of the analysis using the square well model considering joint variation of all constants.

\begin{tabular}{lcl}
\hline Relative variation & Value & $\sigma$ \\
\hline$\frac{\delta \Omega_{B} h^{2}}{\Omega_{B} h^{2}}$ & -0.011 & 0.054 \\
$\frac{\delta \alpha}{\alpha}$ & -0.032 & 0.072 \\
$\frac{\delta\langle v\rangle}{\langle v\rangle}$ & -0.011 & 0.078 \\
\hline
\end{tabular}

Table 7. Results of the analysis using the square well model considering variation of each constant only.

\begin{tabular}{lrl}
\hline Relative variation & Value & $\sigma$ \\
\hline$\frac{\delta \Omega_{B} h^{2}}{\Omega_{B} h^{2}}$ & -0.013 & 0.056 \\
$\frac{\delta \alpha}{\alpha}$ & -0.015 & 0.006 \\
$\frac{\delta\langle v\rangle}{\langle v\rangle}$ & 0.017 & 0.007 \\
\hline
\end{tabular}

probability of $79 \%$ to obtain a worse fit. However, we consider the results of the KS test only indicative, since even though the considered data are independent the residuals are not.

Finally, it is interesting to compare our results with other non-standard BBN models. In particular, a non-standard expansion rate and an electron-neutrino asymmetry were considered by several authors (Barger et al 2003a, Steigman 2005, 2006). While D is more sensitive to the baryon density $\left(\Omega_{b} h^{2}\right)$, the effect of a non-zero $v_{e}$ asymmetry is more strong for ${ }^{4} \mathrm{He}$ than for the other relic nuclides. This is similar to the effect of $\langle v\rangle$ as follows from equations (24) and (25), whereas both $\mathrm{D}$ and ${ }^{4} \mathrm{He}$ are sensible to changes in $\alpha$. In the papers cited above, the authors considered the following cases: (i) adding only one free parameter (either $\Delta N_{v}$ or $\left.\xi_{e}\right)$ and (ii) adding two free parameters $\left(\Delta N_{v}\right.$ and $\left.\xi_{e}\right)$ besides the baryon density. They 
consider the WMAP data from the CMBR together with ${ }^{4} \mathrm{He}$ and $\mathrm{D}$ abundances. In the first case, the results are consistent within $1-\sigma$ with $\xi_{e} \neq 0$ and $\Delta N_{v} \neq 0$. In the second case, the results are consistent within $1-\sigma$ with $\xi_{e}=0$ and $\Delta N_{v}=0$. These results are similar to ours in that considering only one free parameter besides the baryon density is consistent with non-standard physics within $1-\sigma$ whereas considering two free parameters is consistent within $1-\sigma$ with the SBBN model. However, it is important to remind that most of the theories where the fundamental constants may vary in cosmological time scales, predict joint variation of constants.

\section{Conclusions}

In this work, we assumed that the discrepancy between SBBN estimation for ${ }^{4} \mathrm{He}$ and D and their observational data is due to a change in time for the fundamental constants: the Higgs vev $\langle v\rangle$, the fine structure constant $\alpha$. We analysed the dependence of the ${ }^{4} \mathrm{He}$ and $\mathrm{D}$ abundances on these fundamental constants within perturbation theory and on deviations with respect to the mean value of the baryonic density. Furthermore, we compared them with the observational data. We find that varying fundamental constants may not solve, in our case, the discrepancy between the theoretical SBBN and the observed data considered in this work. We hope this work stimulates further research in this interesting subject.

\section{Acknowledgments}

Support for this work is provided by Project G11/G071, UNLP. H Vucetich is partially sponsored by Project 42026-F of CONACyT, Mexico. N Chamoun wishes to acknowledge support from TWAS and CONICET, Argentina

\section{References}

Bahcall J N, Steinhardt C L and Schlegel D 2004 Astrophys. J. 600 520-43

Barger V, Kneller J P, Langacker P, Marfatia D and Steigman G 2003a Phys. Lett. B 569 123-8

Barger V, Kneller J P, Lee H S, Marfatia D and Steigman G 2003b Phys. Lett. B 566 8-18

Barr S M and Mohapatra P K 1988 Phys. Rev. D 38 3011-19

Beane S R and Savage M J 2003 Nucl. Phys. A 713 148-64

Bekenstein J D 1982 Phys. Rev. D 251527

Bergström L, Iguri S and Rubinstein H 1999 Phys. Rev. D 6045005

Bernstein J, Brown L S and Feinberg G 1989 Rev. Mod. Phys. 61 25-39

Bethe H A 1949 Phys. Rev. 76 38-50

Bethe H A and Longmire C 1950 Phys. Rev. 77 647-54

Brax P, van de Bruck C, Davis A C and Rhodes C S 2003 Astrophys. Space Sci. 283 627-32

Burles S and Tytler D 1998a Astrophys. J. 507732

Burles S and Tytler D 1998b Astrophys. J. 499699

Campbell B A and Olive K A 1995 Phys. Lett. B 345 429-34

Chand H, Srianand R, Petitjean P, Aracil B, Quast R and Reimers D 2006 Astron. Astrophys. 451 45-56

Christiansen H R, Epele L N, Fanchiotti H and García Canal C A 1991 Phys. Lett. B $267164-6$

Coc A, Vangioni-Flam E, Descouvemont P, Adahchour A and Angulo C 2004a Tours Symposium on Nuclear Physics V, AIP Conf. Proc. 704 341-50

Coc A, Vangioni-Flam E, Descouvemont P, Adahchour A and Angulo C 2004b Astrophys. J. 600 544-52

Crighton N H M, Webb J K, Ortiz-Gil A and Fernández-Soto A 2004 Mon. Not. R. Astron. Soc. $3551042-52$

Cuoco A, Iocco F, Mangano G, Miele G, Pisanti O and Serpico P D 2004 Int. J. Mod. Phys. A 19 4431-53

Cyburt R H 2004 Phys. Rev. D 70023505

Cyburt R H, Fields B D and Olive K A 2003 Phys. Lett. B 567 227-34

Damour T, Piazza F and Veneziano G 2002a Phys. Rev. Lett. 89081601 
Damour T, Piazza F and Veneziano G 2002b Phys. Rev. D 66046007

Damour T and Polyakov A M 1994 Nucl. Phys. B 423532

Dixit V V and Sher M 1988 Phys. Rev. D 37 1097-1098

Dmitriev V F and Flambaum V V 2003 Phys. Rev. D 67063513

Dmitriev V F, Flambaum V V and Webb J K 2004 Phys. Rev. D 69063506

Esmailzadeh R, Starknam G D and Dimopoulos S 1991 Astrophys. J. 378 504-18

Flambaum V V, Leinweber D B, Thomas A W and Young R D 2004 Phys. Rev. D 69115006

Flambaum V V and Shuryak E V 2002 Phys. Rev. D 65103503

Flambaum V V and Shuryak E V 2003 Phys. Rev. D 67083507

Gleiser M and Taylor J G 1985 Phys. Rev. D 31 1904-10

Grupe D, Pradhan A K and Frank S 2005 Studying the variation of the fine structure constant using emission line multiplets Preprint astro-ph/0504027

Ichikawa K and Kawasaki M 2002 Phys. Rev. D 65123511

Ivanchik A, Petitjean P, Rodriguez E and Varshalovich D 2002 Astron. Lett. 28

Ivanchik A, Petitjean P, Rodriguez E and Varshalovich D 2003 Astrophys. Space Sci. 283 583-8

Ivanchik A, Petitjean P, Varshalovich D, Aracil B, Srianand R, Chand H, Ledoux C and Boisse P 2005 A new constraint on the time dependence of the proton-to-electron mass ratio analysis of the q 0347-383 and q 0405-443 spectra Preprint astro-ph/0507174

Izotov Y and Thuan T 1998 Astrophys. J. 500188

Izotov Y, Thuan T and Lipovetsky V A 1997 Astrophys. J. Suppl. 1081

Kaluza T 1921 Sitzungber. Preuss. Akad. Wiss. K 1966

Kawano L 1992 FERMILAB-PUB-92-004-A

Kirkman D, Tytler D, Suzuki N, O’Meara J M and Lubin D 2003 Astrophys. J. Suppl. Ser. 149 1-28

Klein O 1926 Z. Phys. 37895

Kneller J P and McLaughlin G C 2003 Phys. Rev. D 68103508

Landau S J, Mosquera M E and Vucetich H 2006 Astrophys. J. 637 38-52

Langacker P, Segré G and Strassler M J 2002 Phys. Lett. B 528 121-8

Müller C M, Schäfer G and Wetterich C 2004 Phys. Rev. D 70083504

Maeda K 1988 Mod. Phys. Lett. A 31243

Martínez Fiorenzano A F, Vladilo G and Bonifacio P 2003 Soc. Astron. Ital. Mem. Suppl. 3252

Mukhanov V 2003 Nucleosynthesis without a computer Preprint astro-ph/0303073

Murphy M T, Webb J K and Flambaum V V 2003 Mon. Not. R. Astron. Soc. 345 609-38

Murphy M T, Webb J K, Flambaum V V, Dzuba V A, Churchill C W, Prochaska J X, Barrow J D and Wolfe A M 2001a Mon. Not. R. Astron. Soc. 327 1208-22

Murphy M T, Webb J K, Flambaum V V, Prochaska J X and Wolfe A M 2001b Mon.Not. R. Astron. Soc. 327 1237-43

Nollett K M and Lopez R E 2002 Phys. Rev. D 66063507

Olive K A, Pospelov M, Qian Y Z, Coc A, Cassé M and Vangioni-Flam E 2002 Phys. Rev. D 66045022

Olive K A and Skillman E D 2004 Astrophys. J. 617 29-49

Olive K A and Steigman G 1995 Astrophys. J. Suppl. 9749

O’Meara J M, Tytler D, Kirkman D, Suzuki N, Prochaska J X, Lubin D and Wolfe A M 2001 Astrophys. J. 552718

Overduin J M and Wesson P S 1997 Phys. Rep. 283 303-78

Palma G A, Brax P, Davis A C and van de Bruck C 2003 Phys. Rev. D 68123519

Peimbert M, Peimbert A and Ruiz M 2000 Astrophys. J. 541688

Pettini M and Bowen D V 2001 Astrophys. J. 56041

Quast R, Reimers D and Levshakov S A 2004 Astron. Astrophys. 415 L7-L11

Richard O, Michaud G and Richer J 2005 Astrophys. J. 619 538-48

Romano D, Tosi M, Matteucci F and Chiappini C 2003 Mon. Not. R. Astron. Soc. 346 295-303

Sarkar U 1996 Rep. Prog. Phys. 591493

Schiff L 1968 Quantum Mechanics (New York: McGraw-Hill)

Spergel D N et al 2006 Wilkinson microwave anisotropy probe (WMAP) three year results: implications for cosmology Preprint astro-ph/0603449

Spergel D N et al 2003 Astrophys. J. Suppl. Ser. 148 175-94

Srianand R, Chand H, Petitjean P and Aracil B 2004 Phys. Rev. Lett. 92121302

Steigman G 2005 Phys. Scr. T121 142-6

Steigman G 2006 Int. J. Mod. Phys. E 15 1-35

Wagoner R V 1973 Astrophys. J. 179 343-60

Webb J K, Flambaum V V, Churchill C W, Drinkwater M J and Barrow J D 1999 Phys. Rev. Lett. 82 884-7

Webb J K, Murphy M T, Flambaum V V, Dzuba V A, Barrow J D, Churchill C W, Prochaska J X and Wolfe A M 2001 Phys. Rev. Lett. 87091301 
Weinberg S 1983 Phys. Lett. B 125265

Weinberg S 1990 Phys. Lett. B 251 288-92

Weinberg S 1991 Nucl. Phys. B 3633

Wu Y and Wang Z 1986 Phys. Rev. Lett. 571978

Yoo J J and Scherrer R J 2003 Phys. Rev. D 67043517

Youm D 2001a Phys. Rev. D 63125011

Youm D 2001b Phys. Rev. D 64085011 